\title{
Disentangling hadronic from leptonic emission in the composite SNR G326.3-1.8
}

\author{
J. Devin ${ }^{1}$, F. Acero ${ }^{2}$, J. Ballet ${ }^{2}$, and J. Schmid ${ }^{2}$ \\ ${ }^{1}$ Laboratoire Univers et Particules de Montpellier, Université Montpellier, CNRS/IN2P3, 34095 Montpellier, France \\ e-mail: jdevin.phys@gmail.com \\ 2 Laboratoire AIM, CEA-IRFU/CNRS/Université Paris Diderot, Service d'Astrophysique, CEA Saclay, 91191 \\ Gif sur Yvette, France \\ e-mail: fabio.acero@cea.fr
}

Received 12 March 2018 / Accepted 25 May 2018

\begin{abstract}
Context. G326.3-1.8 (also known as MSH 15-56) has been detected in radio as middle-aged composite supernova remnant (SNR) consisting of an SNR shell and a pulsar wind nebula (PWN) that has been crushed by the SNR reverse shock. Previous $\gamma$-ray studies of SNR G326.3-1.8 revealed bright and extended emission with uncertain origin. Understanding the nature of the $\gamma$-ray emission allows probing the population of high-energy particles (leptons or hadrons), but can be challenging for sources of small angular extent. Aims. With the recent Fermi Large Area Telescope data release Pass 8, which provides increased acceptance and angular resolution, we investigate the morphology of this SNR to disentangle the PWN from the SNR contribution. In particular, we take advantage of the new possibility to filter events based on their angular reconstruction quality.

Methods. We performed a morphological and spectral analysis from $300 \mathrm{MeV}$ to $300 \mathrm{GeV}$. We used the reconstructed events with the best angular resolution (PSF3 event type) to separately investigate the PWN and the SNR emissions, which is crucial to accurately determine the spectral properties of G326.3-1.8 and understand its nature.

Results. The centroid of the $\gamma$-ray emission evolves with energy and is spatially coincident with the radio PWN at high energies $(E>3 \mathrm{GeV})$. The morphological analysis reveals that a model considering two contributions from the SNR and the PWN reproduces the $\gamma$-ray data better than a single-component model. The associated spectral analysis using power laws shows two distinct spectral features, a softer spectrum for the remnant $(\Gamma=2.17 \pm 0.06)$ and a harder spectrum for the PWN $(\Gamma=1.79 \pm 0.12)$, consistent with hadronic and leptonic origin for the SNR and the PWN, respectively. Focusing on the SNR spectrum, we use one-zone models to derive some physical properties, and we find in particular, that the emission is best explained with a hadronic scenario in which the high target density is provided by radiative shocks in H i clouds struck by the SNR.
\end{abstract}

Key words. astroparticle physics - cosmic rays - ISM: supernova remnants - gamma rays: ISM

\section{Introduction}

Supernova remnants (SNRs) and pulsar wind nebulae (PWNe) have long been considered potential sources of Galactic cosmic rays and have therefore been investigated over a wide range of energies. In SNRs, the fast shock wave propagating into the interstellar medium (ISM) or the circumstellar medium is thought to accelerate particles (electrons and protons), which gain energy through first-order Fermi acceleration (Bell 1978); this is also known as the diffusive shock acceleration mechanism (DSA). In core-collapse SNRs, a very fast rotating and highly magnetized pulsar can give rise to a PWN in which electrons and positrons from the pulsar wind are reaccelerated to relativistic energies at a termination shock. These Galactic accelerators have mostly been studied independently, while in case of core-collapse SNRs, the SNR, the PWN, and the pulsar are part of the same object. However, for systems with angular sizes smaller than or comparable to the instrument point-spread function (PSF), it can be difficult to assess the origin of the emission, in particular at $\gamma$-ray energies, where the angular resolution is comparatively much coarser than in the radio and X-ray ranges. Although it can be challenging to understand their origin, these $\gamma$ rays allow probing the population of high-energy particles, such as accelerated electrons interacting with the cosmic microwave background (CMB) or other target photons by inverse Compton (IC) scattering, and also accelerated protons interacting with gas that produce neutral pions that decay into $\gamma$ rays. Morphological studies complementing purely spectral analyses may help identify multiple particleacceleration regions in one object, such as interaction regions with surrounding clouds or the different emissions coming from the SNR and/or the PWN in composite objects.

With an SNR shell and a PWN seen at radio wavelengths, the Galactic SNR G326.3-1.8 is a prototype of the socalled composite SNRs (Mills et al. 1961; Milne et al. 1979). Its distance is estimated between $3.1 \mathrm{kpc}$ (Goss et al. 1972) and $4.1 \mathrm{kpc}$ (Rosado et al. 1996), as established by the $\mathrm{H}_{\mathrm{I}}$ absorption profile and $\mathrm{H} \alpha$ velocity measurements, respectively. Temim et al. (2013) estimated this SNR to be $16500 \mathrm{yr}$ old, with a shock velocity of $500 \mathrm{~km} \mathrm{~s}^{-1}$, expanding in an ISM density of $n_{0}=0.1 \mathrm{~cm}^{-3}$. Figure 1 , obtained from radio observations (Whiteoak \& Green 1996), shows a symmetric SNR shell with $0.3^{\circ}$ radius and a PWN trailing the putative pulsar and likely crushed by the reverse shock of the remnant. Nonthermal radio emission has been reported, with a spectral index of $\alpha=0.34$ for the shell and $\alpha=0.18$ for the nebula, where $S_{v} \propto v^{-\alpha}$ (Dickel et al. 2000). Optical H $\alpha$ filaments were observed in the southwest and northeast parts of the remnant, and they appear to be spatially correlated with the shell (van den Bergh 1979; Dennefeld 1980), indicating neutral 


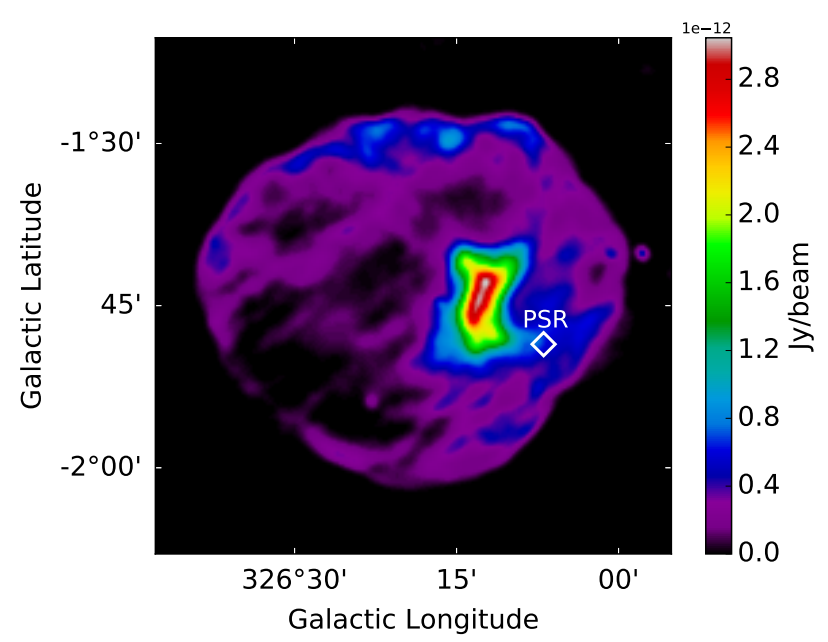

Fig. 1. $843 \mathrm{MHz}$ MOST radio image of SNR G326.3-1.8 (Whiteoak \& Green 1996). The position of the pulsar candidate is represented by a white diamond. The positional uncertainty is much smaller than the marker size.

material at the shock front. The PWN component is highly polarized, with a luminosity of $\mathrm{L}\left(10^{7}-10^{11} \mathrm{~Hz}\right) \sim 5 \times 10^{34} \mathrm{erg} \mathrm{s}^{-1}$ (Dickel et al. 2000). The associated pulsar has not been detected, but Chandra maps have revealed a point source embedded in the X-ray PWN located southwest of the radio nebula (Temim et al. 2013). SNR G326.3-1.8 was also detected in X-rays by ROSAT (Kassim et al. 1993) and ASCA (Plucinsky 1998), showing a complete shell that spatially correlates with the radio SNR, while the width of the PWN in X-rays shrinks near the compact object. At higher energies, previous $\gamma$-ray studies have revealed emission with uncertain origin (Temim et al. 2013), and SNR G326.3-1.8 has only recently been found to be extended with Fermi-LAT data (Acero et al. 2016b).

The latest Large Area Telescope data release Pass 8 (Atwood et al. 2013) allows not only a claim of significant extension of the $\gamma$-ray emission, but also a separate study of the PWN and SNR contributions. This distinction might be crucial for understanding the underlying emission mechanisms and potentially distinguishing between hadronic and leptonic nature of the constituents. In this paper, we briefly describe the latest data release Pass 8 before presenting a morphological study of SNR G326.3-1.8. In particular, we investigate its energy-dependent morphology and model the emission with different templates. We also report a spectral analysis of our best models using two spatial components for the $\gamma$-ray emission and derive physical properties using one-zone models for the SNR spectrum.

\section{Fermi-LAT and Pass 8 description}

The Large Area Telescope (LAT) on board the Fermi satellite is a pair-conversion instrument sensitive to $\gamma$ rays in the energy range from $30 \mathrm{MeV}$ to more than $300 \mathrm{GeV}$.

Since launch in August 2008, the Fermi-LAT event reconstruction algorithm has been progressively upgraded to make use of the increasing understanding of the instrument performance as well as the environment in which it operates. Following Pass 7, released in August 2011, Pass 8 is the latest version of the Fermi-LAT data release (Atwood et al. 2013). The improved reconstruction and classification algorithms result in improvements of the effective area, the PSF, and the energy resolution. One major advance with respect to previous releases is the classification of detected photon events according to their reconstruction quality. The data set is hence divided into types of events with different energy or angular reconstruction qualities. The PSF selection divides the data into four parts: from PSF0 to PSF3, the latter being the quartile with the best angular resolution $\left(68 \%\right.$ containment radius of $0.4^{\circ}$ at $1 \mathrm{GeV}$ compared to $0.8^{\circ}$ without selection). This type of event selection, combined with the large amount of data collected by the LAT since its launch, makes Pass $8 \gamma$ rays a powerful tool to identify and study extended $\gamma$-ray sources.

\section{Data analysis}

We performed a binned analysis using $6.5 \mathrm{yr}$ of data collected from August 4, 2008, to January 31, 2015, within a $10^{\circ} \times 10^{\circ}$ region around the position of SNR G326.3-1.8. Since the object remains significant with $25 \%$ of the data (more than $24 \sigma$ between $300 \mathrm{MeV}$ and $300 \mathrm{GeV}$ ), we took advantage of the new PSF3 selection to limit contamination between the PWN and the SNR components as well as that from the Galactic plane. We selected events between $300 \mathrm{MeV}$ and $300 \mathrm{GeV}$, with a maximum zenith angle of $100^{\circ}$, to reduce the contamination of the bright Earth limb. Time intervals during which the rocking angle of the satellite was more than $52^{\circ}$ were excluded, as were those during which it passed through the South Atlantic Anomaly. We set the pixel size to $0.05^{\circ}$ and divided the whole energy range $(300 \mathrm{MeV}-300 \mathrm{GeV})$ into 30 bins. We used version 10 of the Science Tools (v10r0p5) and the P8R2_V6 instrument response functions (IRFs) with the SOURCE event class for the following analysis ${ }^{1}$. The resulting count map of the $10^{\circ} \times 10^{\circ}$ region centered on the position of the SNR is shown in Fig. 2.

The $\gamma$-ray data around the source were modeled starting with the Fermi-LAT 3FGL source catalog (Acero et al. 2015), complemented by the extended source FGES J1553.8-5325 (Ackermann et al. 2017). We first fit the point sources and extended sources within a $10^{\circ}$ radius (additionally accounting for the most significant sources between $10^{\circ}$ and $15^{\circ}$ ) simultaneously with the Galactic and isotropic diffuse emissions described by the files gll_iem_v06.fits (Acero et al. 2016a) and iso_P8R2_SOURCE_V6_V06_PSF3.txt respectively ${ }^{3}$. We then computed a residual test statistic (TS) map to search for additional sources.

The TS is defined to test the likelihood of one hypothesis $\mathcal{L}_{1}$ (including a source) against the null hypothesis $\mathcal{L}_{0}$ (absence of source), such that

$\mathrm{TS}=2 \times\left(\log \mathcal{L}_{1}-\log \mathcal{L}_{0}\right)$

This can be directly interpreted in terms of significance of hypothesis 1 with respect to the null hypothesis 0 , in which the TS follows a $\chi^{2}$-law with $n$ degrees of freedom for $n$ additional parameters. To evaluate the significance of putative new sources, we computed a $2 \mathrm{D}$ residual TS map that tests the hypothesis of a point source with a generic $E^{-2}$ spectrum against the null hypothesis at each point in the sky. The positions where the TS values exceeded 25 (corresponding to a significance of more than $4 \sigma$ ) were used as seeds to identify $\gamma$-ray sources in addition to the 3FGL. In this way, we iteratively added 11 sources in the $10^{\circ} \times 10^{\circ}$ region, and we fixed their spectral parameters to their

\footnotetext{
1 The Science Tools package and related documentation are distributed by the Fermi Science Support Center at https://fermi .gsfc . nasa. gov/ssc

2 From the Fermi Galactic Extended Source catalog.

3 Available at https://fermi.gsfc.nasa.gov/ssc/data/ access/lat/BackgroundModels.html
} 


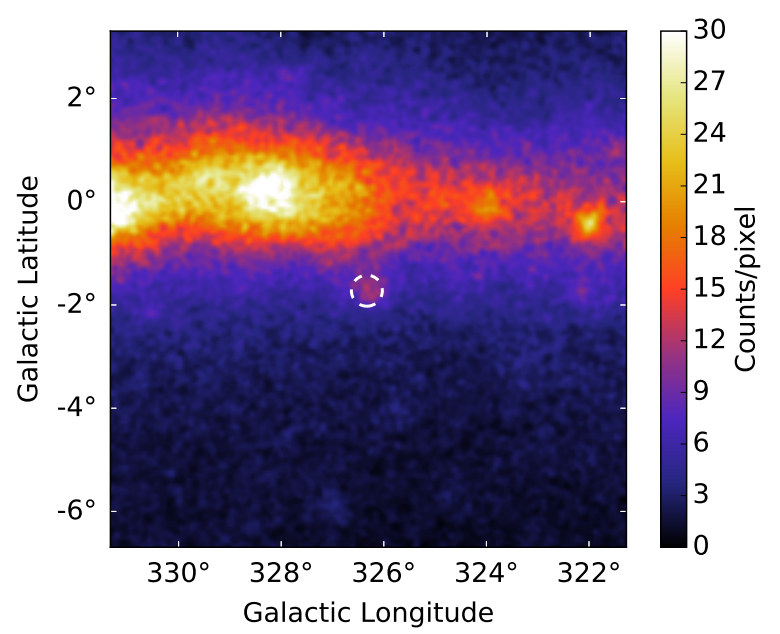

Fig. 2. Count map from $300 \mathrm{MeV}$ to $300 \mathrm{GeV}$ of a $10^{\circ} \times 10^{\circ}$ region centered on the position of the SNR (dashed circle) and using the PSF3 events. The pixel size is $0.05^{\circ}$.

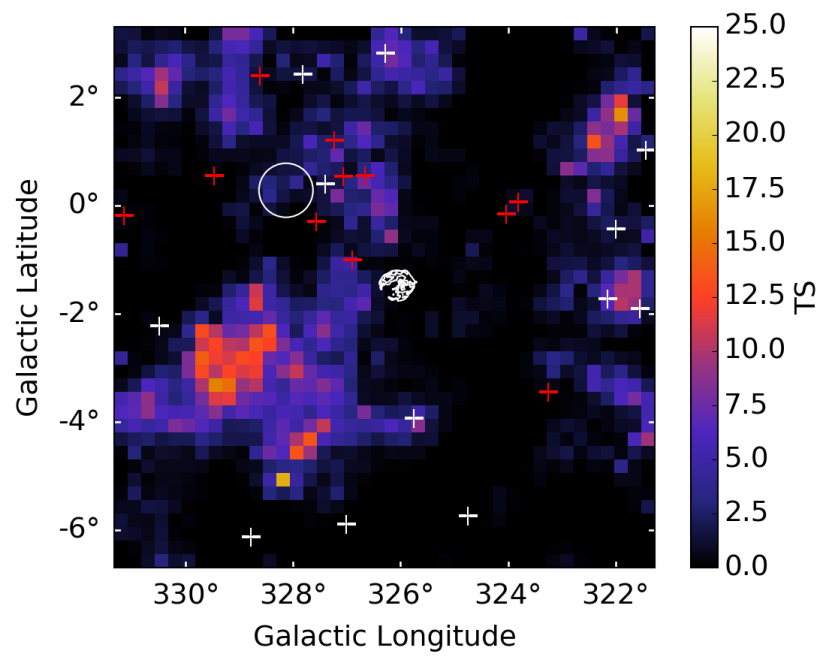

Fig. 3. Residual TS map from $300 \mathrm{MeV}$ to $300 \mathrm{GeV}$ of a $10^{\circ} \times 10^{\circ}$ region centered on the SNR and using the PSF3 events. The pixel size is $0.25^{\circ}$ and the radio contours of the SNR are overlaid in white. The white circle is an FGES extended source. The white crosses are the 3FGL point sources and the red crosses are the sources we added to the model.

best-fit values found with gt like. Figure 3 shows the final residual TS map including all the sources. We note that the apparent diffuse residual emission (for which $\mathrm{TS}_{\max } \approx 17$ ) disappears above $500 \mathrm{MeV}$.

\subsection{Morphological analysis}

\subsubsection{Extension}

The 3FGL catalog compiled by the Fermi-LAT collaboration (Acero et al. 2015) has two point-like sources tentatively associated with the SNR, which we removed for our analysis. Since the creation of the first SNR catalog (Acero et al. 2016b), G326.3-1.8 has been known to show extended $\gamma$-ray emission, and its radius has been determined to be $0.21^{\circ}$ using an extended uniform disk model, somewhat smaller than the $0.31^{\circ}$ radius of the radio shell, but larger than the radio PWN. However, that analysis was based on only 3 years of data and made use of the former Pass 7 data release.
With the latest Pass 8 data and using the PSF3 event type, we revisited the morphology of this SNR to understand the nature of the $\gamma$-ray emission. We started by finding the best position of a point source, modeling its emission as a power law and using the pointlike framework (Kerr 2010) from $300 \mathrm{MeV}$ to $300 \mathrm{GeV}$. Then, we investigated the extension of the $\gamma$-ray emission using a 2D symmetric Gaussian and a disk with a uniform brightness. Table 1 shows the respective best-fit position and extension - if extended - for the different spatial models. The significance of a source extension is expressed in terms of the test statistic $\mathrm{TS}_{\mathrm{ext}}$, where the hypothesis of the best-fit extended spatial model is tested against the null hypothesis of the best-fit point-like source. Given that in both hypotheses the localization of the source is optimized, the extended source model adds one degree of freedom - the source size - with respect to the point-source model. Thus, the significance can be directly interpreted as the square root $\sqrt{\mathrm{TS}_{\mathrm{ext}}}$. As reported in Table 1, the $\gamma$-ray emission is extended with more than $13 \sigma$ confidence level, and the uniform disk radius is found to be $r=0.266^{\circ} \pm 0.012^{\circ}$. Figure 4 (left) shows the best-fit position and extension $(68 \%$ containment radius $r_{68}$ ) for the disk and the Gaussian, plotted in the radio image, with the associated uncertainties. The centroid of each extended model is slightly shifted toward the radio PWN, but is not coincident with its position. No significant residual emission appears in the residual TS map (not shown here) including either the disk or the Gaussian in the model.

\subsubsection{Energy-dependent morphology}

Although the $\gamma$-ray emission can be adequately described with a one-component model, either a disk or a 2D symmetric Gaussian, this slightly contradicts the discovery of a hard pointlike $\gamma$-ray source above $50 \mathrm{GeV}$ (Ackermann et al. 2016) at the location of the PWN, clearly displaced from the center of the SNR shell. To investigate the morphology in more detail, we divided the data into five logarithmically spaced energy bins from $300 \mathrm{MeV}$ to $300 \mathrm{GeV}$ that we subsequently fit individually with pointlike using a 2D symmetric Gaussian. Because the PSF width depends strongly on energy up to $\sim 10 \mathrm{GeV}$ and our energy bins are quite broad (half a decade), we needed to adopt a specific spectral model for the source. We described it with a power law with free spectral index. The normalizations of the source, the Galactic and isotropic diffuse emissions were let free, while the spectral parameters of the other sources were fixed to their best-fit values. Figure 4 (right) depicts the results of the fitting procedure in the individual energy bands. At low energies $(300 \mathrm{MeV}-1 \mathrm{GeV})$, the PSF $\left(r_{68} \sim 0.4^{\circ}\right.$ at $\left.1 \mathrm{GeV}\right)$ is larger than the SNR radius. An extended source of the SNR size is compatible with the data, but is not significantly better than a point source. The associated best-fit position lies outside the radio PWN. Between 1 and $3 \mathrm{GeV}$, the significance of the extension is more than $5 \sigma$ (the values are reported in Fig. 5), and the position of the Gaussian appears to be fairly consistent with the center of the radio SNR. At higher energies (from 3 to $30 \mathrm{GeV}$ ), the $\gamma$-ray morphology is still significantly extended (more than $5 \sigma$ ), and the centroid of the best-fit Gaussian approaches the radio PWN. Above $30 \mathrm{GeV}$, the $\gamma$-ray emission is not significantly extended, and the best-fit position lies inside the radio PWN.

\subsubsection{Building a more detailed model}

This energy-dependent source morphology clearly requires a more detailed investigation beyond a one-component modeling. 
Table 1. Best-fit positions and sizes (radius or sigma) with the associated statistical errors using different spatial models.

\begin{tabular}{lcccccc}
\hline \hline Spatial model & $\mathrm{RA}_{\mathrm{J} 2000}\left(^{\circ}\right)$ & $\operatorname{Dec}_{\mathrm{J} 2000}\left({ }^{\circ}\right)$ & $r$ or $\sigma\left(^{\circ}\right)$ & $r_{68}\left(^{\circ}\right)$ & $\mathrm{TS} \mathrm{TS}_{\mathrm{ext}}$ \\
\hline Point source & $238.167 \pm 0.009$ & $-56.181 \pm 0.008$ & - & - & 689.5 & - \\
Disk & $238.170 \pm 0.012$ & $-56.152 \pm 0.012$ & $0.266 \pm 0.012$ & $0.218 \pm 0.010$ & 866.5 & 177.0 \\
Gaussian & $238.157 \pm 0.013$ & $-56.166 \pm 0.012$ & $0.134 \pm 0.009$ & $0.202 \pm 0.014$ & 863.4 & 173.9 \\
\hline
\end{tabular}

Notes. $r_{68}$ corresponds to the $68 \%$ containment radius of each extended model. The TS and $\mathrm{TS}_{\mathrm{ext}}$ values are also given.
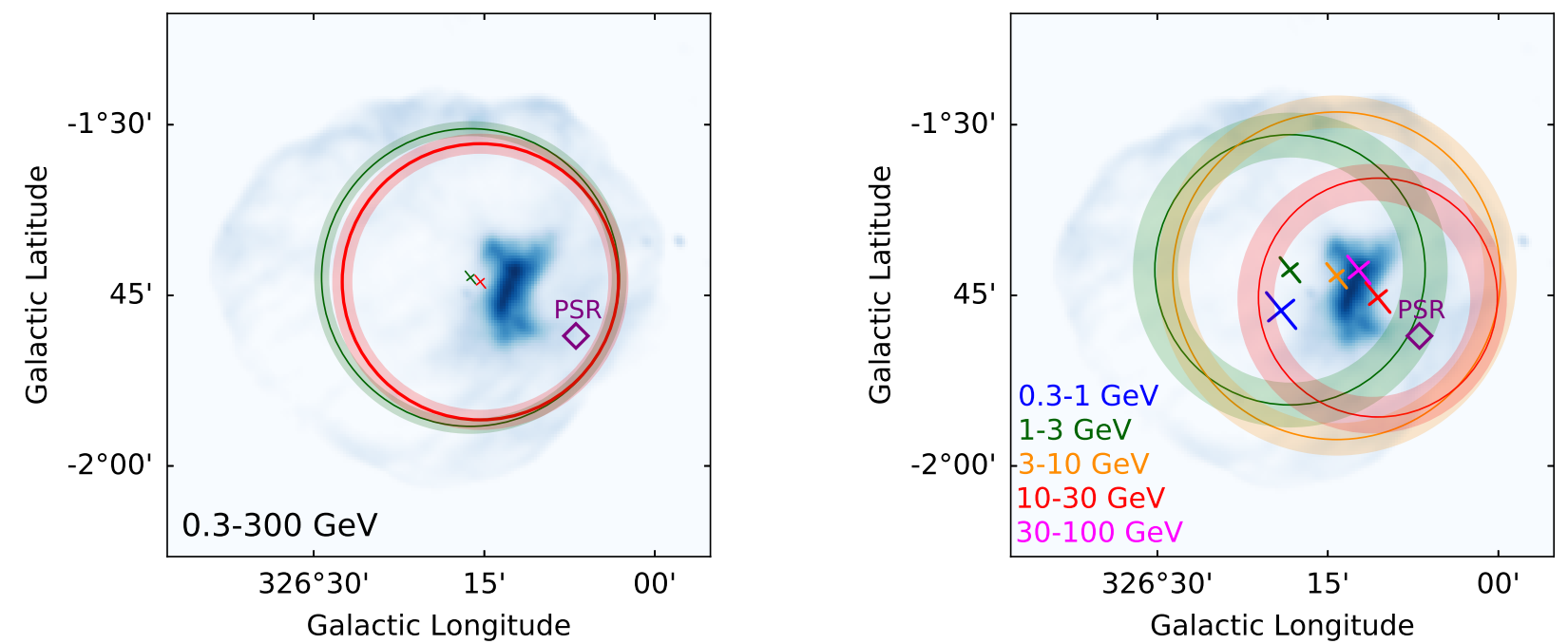

Fig. 4. Left: best-fit extended source from $300 \mathrm{MeV}$ to $300 \mathrm{GeV}$ for a uniform disk hypothesis (green) and a 2D symmetric Gaussian (red), plotted in the MOST radio image. The crosses are the centroid uncertainties $(1 \sigma)$, the solid circles correspond to the $r_{68}$ of the uniform disk, and the Gaussian template and the shaded areas are the $1 \sigma$ error on size. The putative position of the pulsar is represented by a diamond. Right: bestfit Gaussian or point source in five energy bands. The crosses are the centroid and the position uncertainties $(1 \sigma)$. If the source is significantly extended (see Fig. 5), the $r_{68}$ of the Gaussian is depicted by a solid circle with the associated errors (shaded areas), otherwise the best-fit point source is represented.

Since the PSF below $1 \mathrm{GeV}$ is not small enough to resolve the $\mathrm{SNR}$, the following morphological analysis uses data between 1 and $300 \mathrm{GeV}$.

Electrons and positrons, accelerated in the PWN, that radiate by synchrotron emission are expected to also radiate in the $\mathrm{GeV}$ band by IC scattering on photon fields. Since this SNR is relatively young, particles are still confined inside the PWN. Temim et al. (2013) estimated the magnetic field to be $B_{\mathrm{PWN}} \approx 34 \mu \mathrm{G}$ when the SNR has already begun to significantly compress the PWN at an age of $19000 \mathrm{yr}$. The emission seen in the radio band should track the older accelerated electrons, and we expect that the extension of the $\gamma$-ray emission should not exceed the radio emission. We thus modeled the $\gamma$-ray emission from the PWN using its radio template (see Fig. 6, left panel), knowing that the magnetic field spatial distribution inside the PWN should only moderately impact our model given the small size of the PWN compared to the Fermi-LAT PSF.

Since the best-fit position of the $\gamma$-ray emission is consistent with that of the PWN at high energies, we first assumed that the $\gamma$-ray emission comes only from the PWN. We modeled its spectrum as a power law and performed a likelihood fit where the spectral parameters of the PWN, those of the nearest point source, and the Galactic and isotropic diffuse emissions were free during the fit. The spectral parameters of the other sources were fixed to their best-fit values since they lie farther than $\sim 2^{\circ}$ away from G326.3-1.8. The fit gives a TS value of TS $=593.4$, as reported in Table 2 , with the number of additional free parameters compared to the model without source.

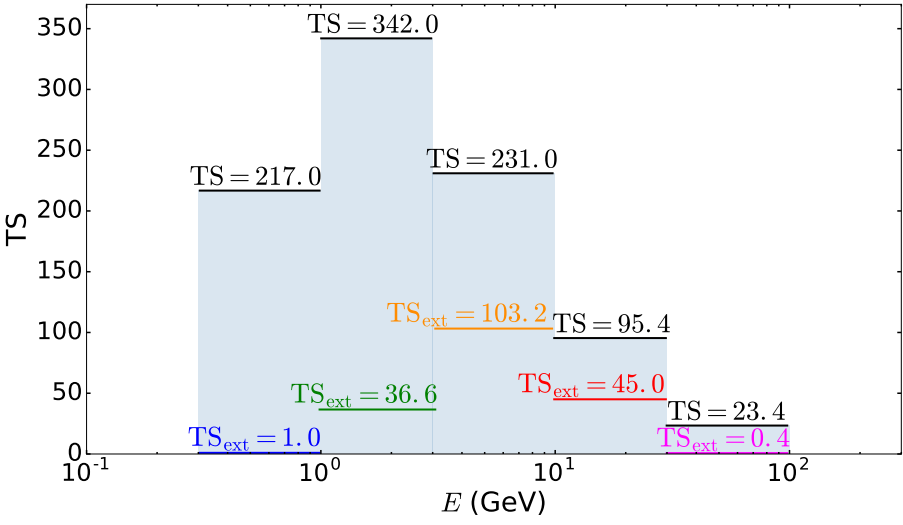

Fig. 5. Test statistic of the source (black bars) and of extension (colored bars) for the best-fit Gaussian in individual energy bands.

Figure 7 (left) depicts the $1^{\circ} \times 1^{\circ}$ residual TS map from 1 to $300 \mathrm{GeV}$ obtained by fixing the spectral parameters of the radio PWN and the nearest point source to their best-fit values. The TS map tests a putative point source. It shows qualitatively where signal is missing, and extended emission can only be more significant than the peak of the TS map. The maximum TS value of the map is TS $\approx 60$, indicating that this residual emission is clearly significant. The radio template of the PWN is thus not sufficient to describe the data. This confirms our previous results in Sect. 3.1.2, which show that the emission below $3 \mathrm{GeV}$ lies outside of the radio contours of the PWN. 

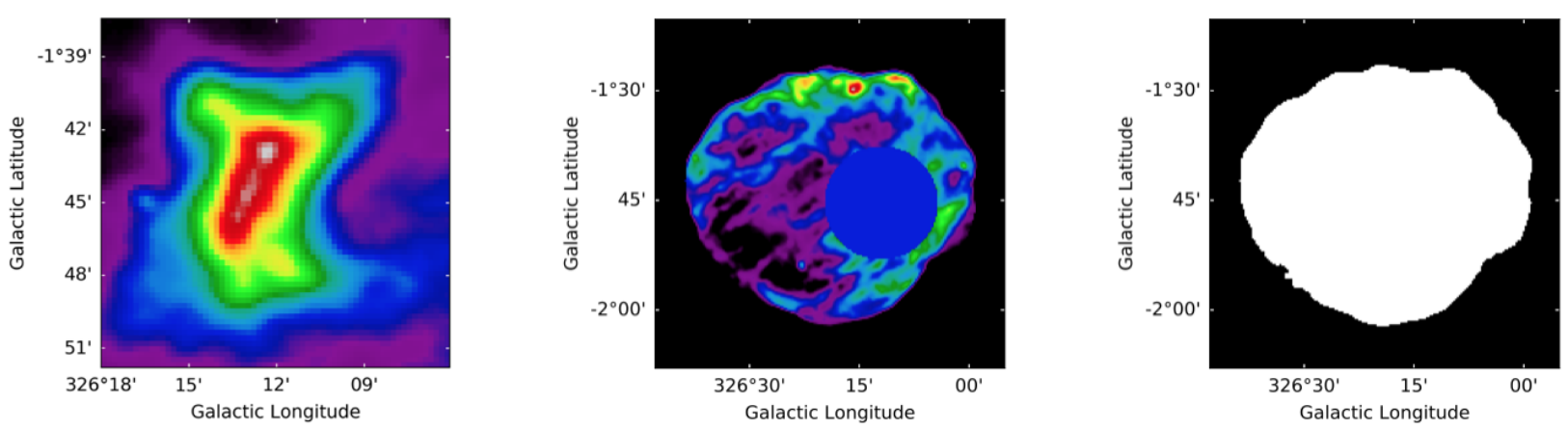

Fig. 6. Templates used in this analysis: left: radio PWN, center: the radio SNR with the PWN contribution replaced by the average value of the radio emission around it (blue disk), right: SNR mask derived from the SNR radio template and filled homogeneously. The radio PWN (left) does not have the same scale as the two other templates.
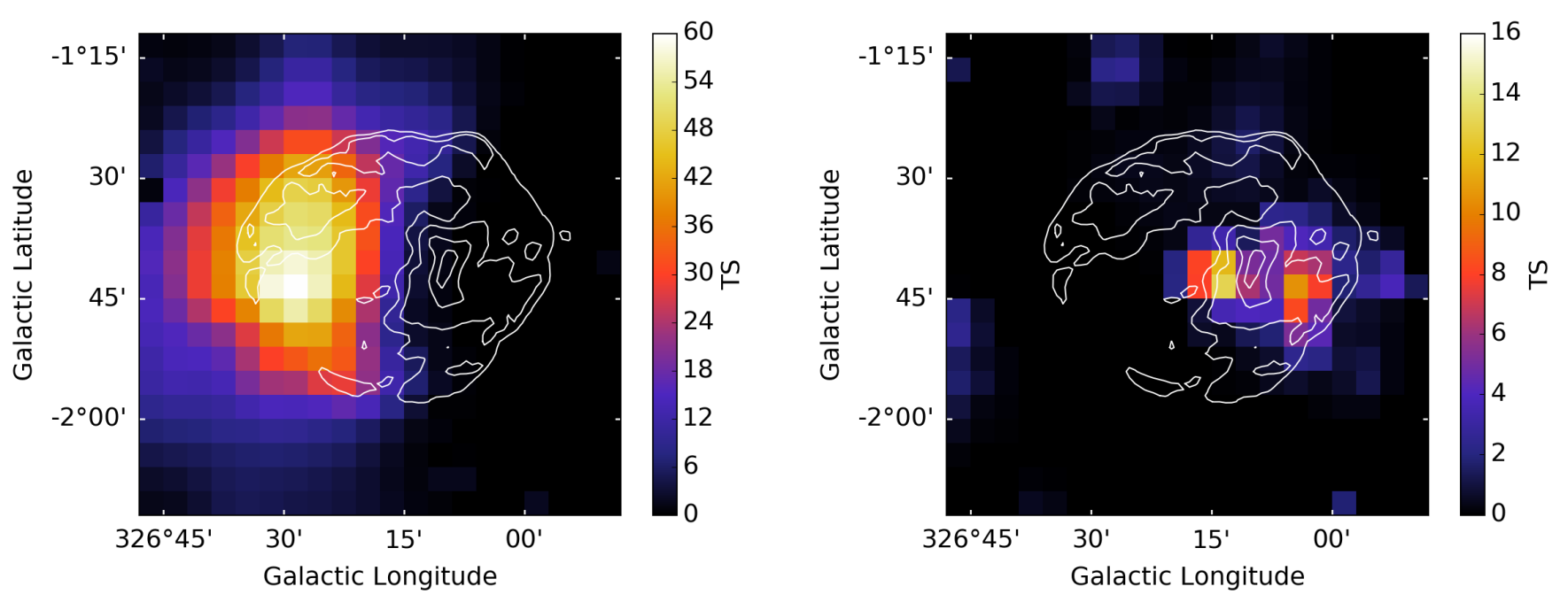

Fig. 7. Residual $1^{\circ} \times 1^{\circ} \mathrm{TS}$ maps from $1 \mathrm{GeV}$ to $300 \mathrm{GeV}$ when we use only the radio PWN (left) or the SNR mask (right) to describe the $\gamma$-ray emission (note that the TS bars do not have the same scale). The pixel size is $0.05^{\circ}$, and the radio contours of the whole SNR are overlaid in white.

Table 2. TS values for different spatial models fit from $1 \mathrm{GeV}$ to $300 \mathrm{GeV}$.

\begin{tabular}{lccc}
\hline \hline Spatial models & TS & $N_{\text {dof }}$ & TS $_{\text {PWN }}$ \\
\hline Radio PWN & 593.4 & 2 & - \\
\hline Point source & 503.3 & 4 & - \\
Point source + radio PWN & 661.4 & 6 & 158.1 \\
\hline Disk & 681.8 & 5 & - \\
Disk + radio PWN & 694.8 & 7 & 13.0 \\
\hline Radio SNR & 667.3 & 2 & - \\
Radio SNR + radio PWN & 683.0 & 4 & 15.7 \\
\hline SNR mask & 670.3 & 2 & - \\
SNR mask + radio PWN & 696.4 & 4 & 26.1 \\
\hline
\end{tabular}

Notes. The corresponding number of degrees of freedom is also given $\left(N_{\text {dof }}\right)$. TS $S_{\text {PWN }}$ quantifies the improvement of the fit when adding the PWN component to each of the one-component models.

To model the contribution of an additional component that seems to give rise to the low-energy part, we tested several templates using first a simple disk component and then physically motivated templates (derived from the radio map of the SNR). We first used the pointlike framework to find the best position and extension of an additional source, described by a disk, when the PWN was already included in the model. The fit localizes the position near the center of the $\mathrm{SNR}$ at $\mathrm{RA}_{\mathrm{J} 2000}=238.169^{\circ} \pm$ $0.013^{\circ}$ and $\operatorname{Dec}_{\mathrm{J} 2000}=-56.133^{\circ} \pm 0.014^{\circ}$ with a radius $r=$ $0.295^{\circ} \pm 0.013^{\circ}$, similar to the radio extension of the SNR $\left(0.31^{\circ}\right)$. The significance of the extension is $5.8 \sigma\left(\mathrm{TS}_{\mathrm{ext}}=33.4\right)$, calculated with the TS value of the model including the point source and the radio PWN (reported in Table 2). This rules out the hypothesis that a point-like source is responsible for the additional emission, such as an active galactic nucleus behind the SNR.

We furthermore used the radio observations to derive two other templates for the SNR. First, we used the radio map and replaced the contribution of the PWN by the average value of the radio emission around it (labeled "radio SNR"; see Fig. 6, center). Based on this, we also created another template, following the radio shock and filled homogeneously (called here "SNR mask"; see Fig. 6, right).

For all components, the $\gamma$-ray emission is described by a power law, and the free spectral parameters are those of the components, the nearest point source, and those of the Galactic and isotropic diffuse emissions. The results from our maximum likelihood fit are given in Table 2 with the numbers of free parameters associated with the models (spectral and/or spatial).

First, the TS values obtained using the one-component models (disk, radio SNR or SNR mask) alone are clearly higher than the value obtained using only the radio PWN, which indicates that the fit prefers a more extended model than the radio PWN. For com- 
Table 3. Results from our maximum likelihood fit between $300 \mathrm{MeV}$ and $300 \mathrm{GeV}$ with the associated statistical errors.

\begin{tabular}{l|c|cc|cc}
\hline \hline Values from the fit & Disk only & Radio PWN & Disk & Radio PWN & SNR mask \\
\hline$\Phi\left(\mathrm{ph} \mathrm{cm}^{-2} \mathrm{~s}^{-1}\right) \times 10^{-8}$ & $2.70 \pm 0.15$ & $0.23 \pm 0.11$ & $2.43 \pm 0.23$ & $0.33 \pm 0.11$ & $2.31 \pm 0.23$ \\
$\Gamma$ & $2.07 \pm 0.04$ & $1.74 \pm 0.15$ & $2.16 \pm 0.06$ & $1.79 \pm 0.12$ & $2.17 \pm 0.06$ \\
\hline
\end{tabular}

Notes. $\Phi$ is the integrated flux and $\Gamma$ is the spectral photon index.
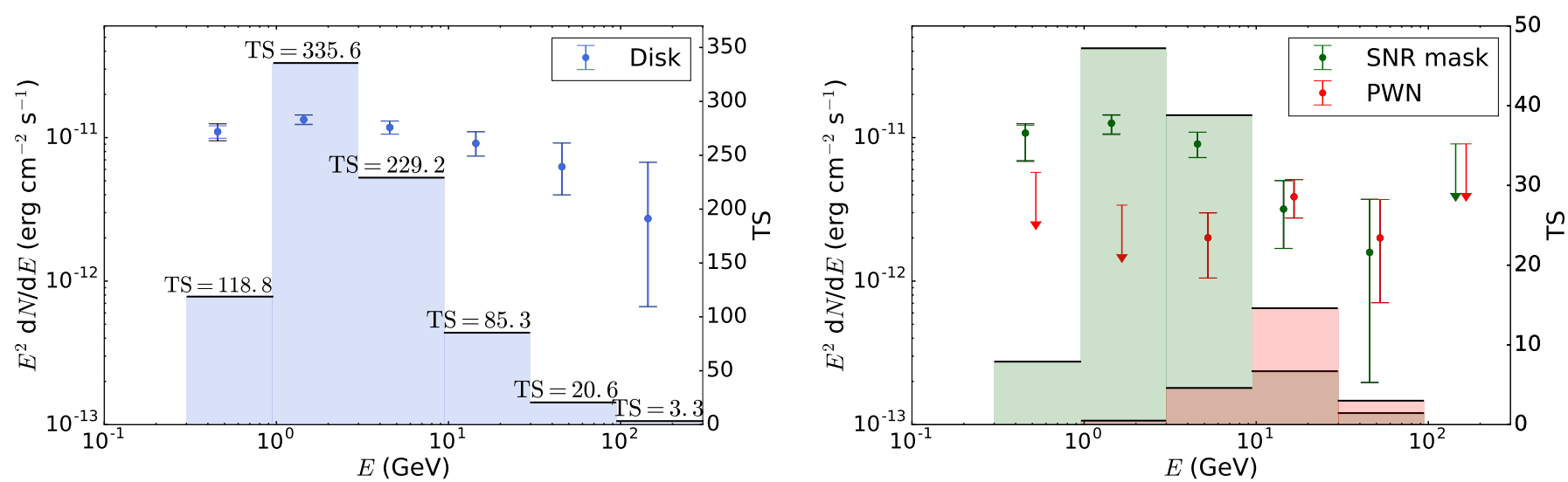

Fig. 8. SED (data points) and TS values (shaded areas) in individual energy bands using the uniform disk model (left) and using the SNR mask and the radio PWN model (right). The colored bars are the statistical uncertainties, and the black bars correspond to the quadratic sums of statistical and systematic errors (the difference is only visible in the first energy band). The systematic errors are calculated using eight alternative Galactic diffuse emission models.

parison, Fig. 7 (right) depicts the residual TS map when only the SNR mask is included in the model, showing a residual emission coincident with the position of the PWN. In terms of test statistic, when adding a second component, the model with one component becomes the null hypothesis to test the significance of the second component. In Table 2 we compare the TS values obtained with each of the one-component models to the two-component models and test the improvement of the fit when the radio template of the PWN is added. The difference $\mathrm{TS}_{\mathrm{PWN}}$ can be converted into a significance since in the null hypothesis (no PWN emission) it behaves as a $\chi^{2}$-law with two degrees of freedom.

For all our extended models, the significance of adding the PWN lies between 3 and $4 \sigma$, and the maximum TS values are obtained for the model including the radio PWN with either the disk or the SNR mask. In terms of significance, our best model involves the radio PWN and the SNR mask since it requires fewer free parameters during the fit than the disk, whose spatial components have been optimized. The lower TS value using the two radio templates (for the SNR and the PWN) indicates that the $\gamma$-ray emission does not entirely follow the synchrotron distribution and the fit prefers a more homogeneous structure for the shell, keeping in mind that this conclusion depends on the model we chose for the PWN.

\subsection{Spectral analysis}

To understand the underlying emission processes, we performed a spectral analysis from $300 \mathrm{MeV}$ to $300 \mathrm{GeV}$ using our best models of the previous section: the disk alone, and the radio PWN with either the SNR mask or the disk. Here the $\gamma$-ray emissions are still described with power laws since other spectral representations did not improve the fit. We did not take into account the energy dispersion (this induces a bias $\sim-5 \%$ on flux ${ }^{4}$ ).

\footnotetext{
4 See https://fermi.gsfc.nasa.gov/ssc/data/analysis/ documentation/Pass8_edisp_usage.html
}

Using gtlike, we performed a maximum likelihood fit leaving the same spectral parameters free as before. Table 3 reports the results obtained from the fit.

When we use the disk alone to describe the $\gamma$-ray emission, the photon index is found to be close to 2. Using differentiated models for the PWN and the SNR, the fit leads to a spectral separation between the two components: a softer spectrum for the remnant $(\Gamma \approx 2.16$ using the disk and $\Gamma \approx 2.17$ using the SNR mask) and a harder spectrum for the nebula $(\Gamma \approx 1.74$ and $\Gamma \approx 1.79$ ). The choice of the model for the remnant (either the disk or the SNR mask) has a very weak effect on the spectral study.

To compute the spectral energy distribution (SED), we divided the whole energy range $(300 \mathrm{MeV}-300 \mathrm{GeV})$ into six bins and imposed a TS threshold of 1 per energy bin for the flux calculation; otherwise, an upper limit was calculated. In each bin, the photon indexes of the sources of interest were fixed to 2 to avoid any dependence on the spectral models. The fluxes of the PWN and the SNR components were let free during the fit, as were those of the Galactic and isotropic diffuse emissions. All other sources were fixed to their best global model.

Figure 8 shows the SED of the uniform disk (left) and the SED of the best-fit two-component model (right) using the radio PWN and the SNR mask. The colored error bars represent the statistical errors, while the quadratic sums of the statistical and systematic errors (calculated using eight alternative Galactic diffuse emission models as explained in the first Fermi-LAT supernova remnant catalog, Acero et al. 2016b) are represented with black horizontal bars. The systematic errors are never dominant and are comparable to the statistical errors only in the first band. We note that the effective area uncertainty also induces systematic errors ( $10 \%$ between $100 \mathrm{MeV}$ and $100 \mathrm{GeV})$. These SEDs clearly emphasize that two distinct morphologies give rise to two distinct spectral signatures, while the different emissions 
seem to be mixed when we use a single-component model. The TS values in each energy bin highlight the different contributions of the two components: at low energy $(E<10 \mathrm{GeV})$, the emission is dominated by the SNR, while the contribution of the PWN becomes important above $10 \mathrm{GeV}$, bringing out the spatial and spectral distinctions between these two nested objects.

\section{Results and discussion}

For this entire section we assume that the distance to the SNR is $4.1 \mathrm{kpc}$ (Temim et al. 2013).

\subsection{SNR spectrum}

To understand the observed $\gamma$-ray spectrum of the SNR, we performed multiwavelength modeling using the one-zone models provided by the naima package (Zabalza 2015). From Dickel et al. (2000), we took the five radio flux measurements of the shell. As there is no associated synchrotron emission in the X-ray domain, we used the ROSAT thermal flux reported by Kassim et al. (1993) as an upper limit. We also used the TeV upper limit derived from H.E.S.S. with $14 \mathrm{~h}$ of observational live time and assuming a photon index of 2.3 (H.E.S.S. Collaboration et al. 2018).

Assuming the Sedov phase, we derived the kinetic energy released by the supernova:

$\frac{E_{\mathrm{SN}}}{10^{51} \mathrm{erg}}=R_{12.5}^{5} \times\left(\frac{n_{0}}{\mathrm{~cm}^{-3}}\right) \times t_{4}^{-2}=0.5$

where $R_{12.5}=R /(12.5 \mathrm{pc})$ and $t_{4}=t /(10000 \mathrm{yr})$ taking $R=21 \mathrm{pc}$, $t=16500 \mathrm{yr}$ and $n_{0}=0.1 \mathrm{~cm}^{-3}$ for a distance of $4.1 \mathrm{kpc}$. We took the inputs from Temim et al. (2013) but did not derive the same explosion energy. Here we used the common values of $\xi=2.026$ (for $\gamma=5 / 3$ ) and $\rho_{0}=1.4 m_{\mathrm{H}} n_{0}$ in the usual Sedov equation: $R^{5}=\xi\left(E_{\mathrm{SN}} / \rho_{0}\right) t^{2}$.

The explosion energy and age depend on the distance, which is still uncertain. In addition, the density and temperature (the latter provides the shock speed estimate) were derived from a small region south of the SNR, and it is not yet clear whether this region is representative of the rest of the SNR. (A Large Program with XMM-Newton on G326.3-1.8 is currently ongoing and will provide more constraints on the thermal emission across the SNR). In this regard, the multiwavelength modeling presented in this section is not to be viewed as a precise measurement of the properties of the accelerated particles, but rather as showing that a simple self-consistent model can reproduce the observations.

We describe the electron population as a broken powerlaw spectrum with spectral indexes $\Gamma_{\mathrm{e}, 1} / \Gamma_{\mathrm{e}, 2}$ with an exponential cutoff. The break at energy $E_{\mathrm{b}}$ is assumed to be due to cooling, therefore we set $\Gamma_{\mathrm{e}, 2}=\Gamma_{\mathrm{e}, 1}+1$, while the cutoff defines the maximum attainable energy of the particles $E_{\text {max,e. }}$. The proton spectrum is described as a power law with spectral index $\Gamma_{\mathrm{p}}$ with an exponential cutoff $E_{\text {max,p. }}$. We consider by default the CMB as the only photon seed for IC scattering in our models.

\subsubsection{Leptonic scenario}

We first investigated the leptonic scenario, for which we varied the values of the magnetic field $B$, the total energy budget in electrons $W_{\mathrm{e}}$ and protons $W_{\mathrm{p}}$, and the break and maximum energy of the particles. Figure 9 (left) shows one of the combinations that simultaneously fits the radio and the $\gamma$-ray data. Since this solution is not unique, we report in Table 4 the range of permitted values of these parameters. For clarity, we fixed the total energy in protons to $W_{\mathrm{p}}=5 \times 10^{49} \mathrm{erg}$ (corresponding to $10 \%$ of $E_{\mathrm{SN}}$ ), and we report the range of permitted values of the electron-proton ratio $K_{\mathrm{e}-\mathrm{p}}$. Since the maximum energy of protons is always higher than that of electrons, which suffer synchrotron losses, we used the maximum value of $E_{\text {max,e }}$ as a lower limit for $E_{\max , \mathrm{p}}$.

To reproduce the radio spectral shape, we needed a hard index for the electrons $\Gamma_{\mathrm{e}, 1}=1.8$ (taking thus $\Gamma_{\mathrm{e}, 2}=2.8$ ), while we kept $\Gamma_{\mathrm{p}}=2$ because we lacked observational constraints. For a $B$ field between 10 and $20 \mu \mathrm{G}$, the $\gamma$-ray data can only be explained if the total energy in electrons reaches $W_{\mathrm{e}}=(2.5-7) \times$ $10^{49} \mathrm{erg}$, which is clearly unreasonable since that requires a $K_{\mathrm{e}-\mathrm{p}}$ between 0.5 and 1.4. If in order to reduce $W_{\mathrm{e}}$, we increase $B$ to higher values than expected for the compressed ISM, the $\gamma$-ray data cannot be fit and the IC spectrum lies one order of magnitude below the data. Even if infrared and optical photon fields with an energy density $0.26 \mathrm{eV} \mathrm{cm}^{-3}$ each (the same as the CMB value, which is a reasonable estimate $100 \mathrm{pc}$ below the Galactic plane) are added, an unrealistically large $W_{\mathrm{e}}$ is still required to fit the $\gamma$-ray data.

Another inconsistency of that model is that the values of $E_{\mathrm{b}}$, $E_{\text {max,e }}$ and $E_{\text {max,p }}$ reported in Table 4 are not consistent with the magnetic field. Following Parizot et al. (2006), we used the synchrotron loss time

$\tau_{\text {sync }}=\left(1.25 \times 10^{3}\right) \times E_{\mathrm{TeV}}^{-1} B_{100}^{-2} \mathrm{yr}$

and the acceleration timescale

$t_{\mathrm{acc}}=30.6 \times \frac{3 r^{2}}{16(r-1)} \times k_{0}(E) \times E_{\mathrm{TeV}} B_{100}^{-1} u_{\mathrm{sh}, 3}^{-2} \quad \mathrm{yr}$,

with $r$ being the shock compression ratio, $k_{0}$ the ratio between the mean free path and the gyroradius, $B_{100}$ and $u_{\mathrm{sh}, 3}$ the magnetic field and the shock velocity in units of $100 \mu \mathrm{G}$ and $1000 \mathrm{~km} \mathrm{~s}^{-1}$, respectively. $k_{0} \geq 1$ can be interpreted as the ratio of the total magnetic energy density to that in the turbulent field $\left(B_{\text {tot }}^{2} / B_{\text {turb }}^{2}\right)$ and $k_{0} \approx 1$ has been found for young SNRs (Uchiyama et al. 2007). For evolved systems, we expect the turbulent magnetic field to be smaller than the large-scale component (so that $k_{0}>1$ ), and we adopted $k_{0}=10$ here for the highest-energy electrons. Taking $r=4$, we thus calculated $E_{\mathrm{b}}$ (equating $\left.\tau_{\text {sync }}=t_{\text {age }}\right), E_{\text {max,e }}\left(t_{\text {acc }}=\right.$ $\left.\min \left\{\tau_{\text {sync }}, t_{\text {age }}\right\}\right)$ and $E_{\text {max,p }}\left(t_{\text {acc }}=t_{\text {age }}\right)$. For $B=20 \mu \mathrm{G}$, we obtained $E_{\mathrm{b}}=1.9 \mathrm{TeV}, E_{\mathrm{max}, \mathrm{e}}=2.3 \mathrm{TeV}$ and $E_{\mathrm{max}, \mathrm{p}}=2.7 \mathrm{TeV}$. Figure 9 (right) shows the corresponding spectrum, which implies an IC cutoff at a too high energy and does not fit the $\gamma$-ray data.

One noteworthy aspect of this source concerns the difficulty to explain its very high radio flux (114 Jy at $1 \mathrm{GHz}$, Dickel et al. 2000). High radio fluxes are also found in middle-aged SNRs that interact with molecular clouds, such as W44 (230 Jy at $1 \mathrm{GHz}$, Castelletti et al. 2007) and IC 443 (160 Jy at $1 \mathrm{GHz}$, Milne 1971), where the highly compressed gas increases the synchrotron emission. The particularly high total energy required in electrons to reproduce the SNR spectrum and the impossibility to fit the data with consistent values rule out a leptonic origin of the $\gamma$-ray emission and led us to investigate the hadronic scenario.

\subsubsection{Hadronic scenario}

van den Bergh (1979) has reported $\mathrm{H} \alpha$ emission in the northeast and southwest regions of the SNR (see Fig. 10, left panel) and 
Table 4. Required physical parameters to model the radio and the $\gamma$-ray data coming from the shell for the leptonic and hadronic scenarios.

\begin{tabular}{|c|c|c|c|c|c|c|c|c|c|c|}
\hline Scenario & Model & $B(\mu \mathrm{G})$ & $W_{\mathrm{p}}(\mathrm{erg})$ & $K_{\mathrm{e}-\mathrm{p}}$ & $\Gamma_{\mathrm{e}, 1} / \Gamma_{\mathrm{e}, 2}$ & $E_{\mathrm{b}}(\mathrm{TeV})$ & $E_{\max , \mathrm{e}}(\mathrm{TeV})$ & $\Gamma_{\mathrm{p}}$ & $E_{\max , \mathrm{p}}(\mathrm{TeV})$ & $n_{0}\left(\mathrm{~cm}^{-3}\right)$ \\
\hline Leptonic & $\begin{array}{l}\text { fit } \\
\text { fit } \\
\text { consistent }\end{array}$ & $\begin{array}{l}10 \\
20 \\
20\end{array}$ & $\begin{array}{l}5 \times 10^{49 *} \\
5 \times 10^{49 *} \\
5 \times 10^{49 *}\end{array}$ & $\begin{array}{l}1.4 \\
0.5 \\
0.5\end{array}$ & $\begin{array}{l}1.8 / 2.8 \\
1.8 / 2.8 \\
1.8 / 2.8\end{array}$ & $\begin{array}{c}(0.15-0.35) \\
(0.4-0.9) \\
1.9^{*}\end{array}$ & $\begin{array}{c}(0.4-1.1) \\
(0.9-1.5) \\
2.3^{*}\end{array}$ & $\begin{array}{l}2^{*} \\
2^{*} \\
2^{*}\end{array}$ & $\begin{array}{c}\left(\geq E_{\max , \mathrm{e}}\right) \\
\left(\geq E_{\max , \mathrm{e}}\right) \\
2.7^{*}\end{array}$ & $\begin{array}{l}0.1^{*} \\
0.1^{*} \\
0.1^{*}\end{array}$ \\
\hline 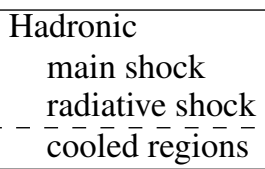 & $\begin{array}{l}\text { consistent } \\
\text { consistent }\end{array}$ & $\begin{array}{c}10 \\
\frac{13.6^{*}}{158^{*}}\end{array}$ & $\begin{array}{l}5 \times 10^{49} \\
\overline{1} . \overline{9} \times \overline{1} 0^{49}\end{array}$ & $\begin{array}{l}0.03 \\
\overline{0} . \overline{0} 3\end{array}$ & $\begin{array}{c}2 / 3^{*} \\
-\overline{1.8 / 2.8}\end{array}$ & $\begin{array}{c}1.4^{*} \\
-\overline{0} . \overline{0} 2^{*}\end{array}$ & $\begin{array}{c}1.4^{*} \\
\overline{0} . \overline{0 .} \overline{8}^{*}\end{array}$ & $\begin{array}{c}2^{*} \\
-2^{*}\end{array}$ & $\begin{array}{c}1.4^{*} \\
-\overline{0.08^{*}}\end{array}$ & $\begin{array}{c}0.1^{*} \\
1.88^{*} \\
88.3^{*}\end{array}$ \\
\hline
\end{tabular}

Notes. The parentheses indicate the range of permitted values, while the asterisks indicate the fixed values in our analysis. The other values (without parentheses and asterisks) are adjusted by hand. In the two leptonic scenarios in which the break energy $E_{\mathrm{b}}$ and maximum energy $E_{\mathrm{max}, \mathrm{e}}$ of the electrons are fit, they are not consistent with the magnetic field if they are due to synchrotron cooling. In all other (consistent) models, they are calculated following Parizot et al. (2006). For the hadronic scenario, the first and the second line correspond to the properties of the main shock $\left(u_{\mathrm{sh}}=500 \mathrm{~km} \mathrm{~s}^{-1}\right)$ and the radiative shock $\left(u_{\mathrm{sh}, \mathrm{cl}}=150 \mathrm{~km} \mathrm{~s}^{-1}\right)$, respectively. The density and the magnetic field in the last line are those of the downstream cooled regions.
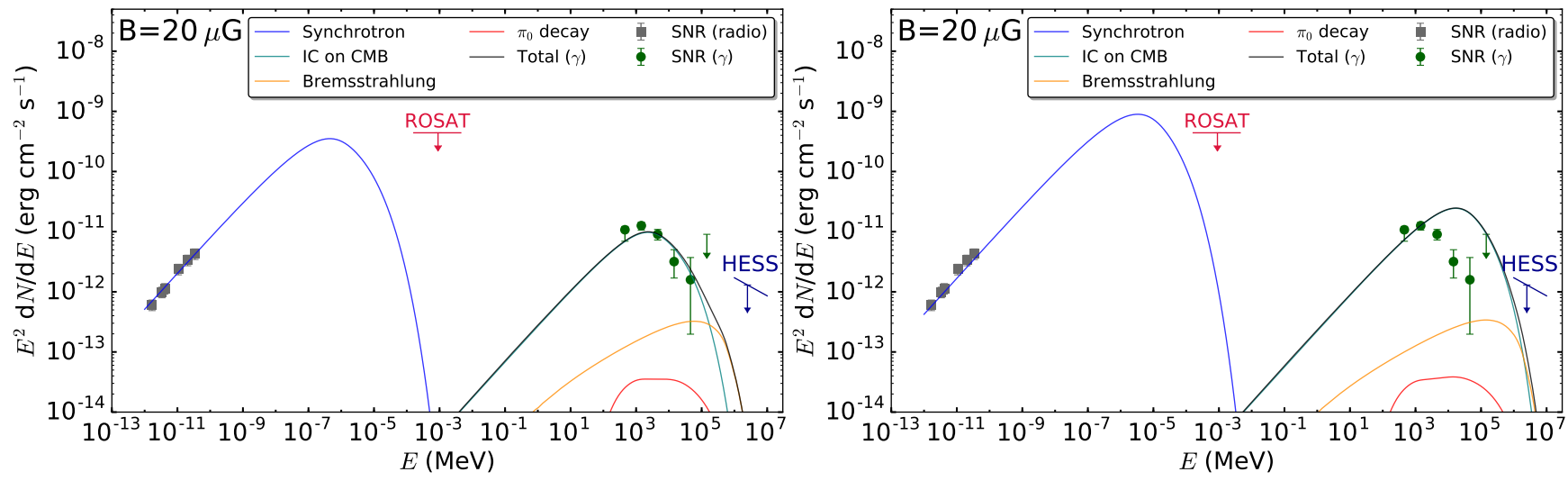

Fig. 9. Multiwavelength modeling of the SNR spectrum in the leptonic scenario. The radio points are extracted from Dickel et al. (2000), the ROSAT and the H.E.S.S. upper limits come from Kassim et al. (1993) and H.E.S.S. Collaboration et al. (2018) respectively. Left: parameters are free to vary, and the values are $E_{\mathrm{b}}=600 \mathrm{GeV}, E_{\mathrm{max}, \mathrm{e}}=1 \mathrm{TeV}, E_{\mathrm{max}, \mathrm{p}}=1 \mathrm{TeV}, W_{\mathrm{p}}=5 \times 10^{49} \mathrm{erg}$, and $K_{\mathrm{e}-\mathrm{p}}=0.5$. The spectral index of the electrons before and after the break is $\Gamma_{\mathrm{e}, 1}=1.8$ and $\Gamma_{\mathrm{e}, 2}=2.8$, respectively. Right: same as in the left panel, but with values consistent with the magnetic field: $E_{\mathrm{b}}=1.9 \mathrm{TeV}, E_{\mathrm{max}, \mathrm{e}}=2.3 \mathrm{TeV}$, and $E_{\mathrm{max}, \mathrm{p}}=2.7 \mathrm{TeV}$.
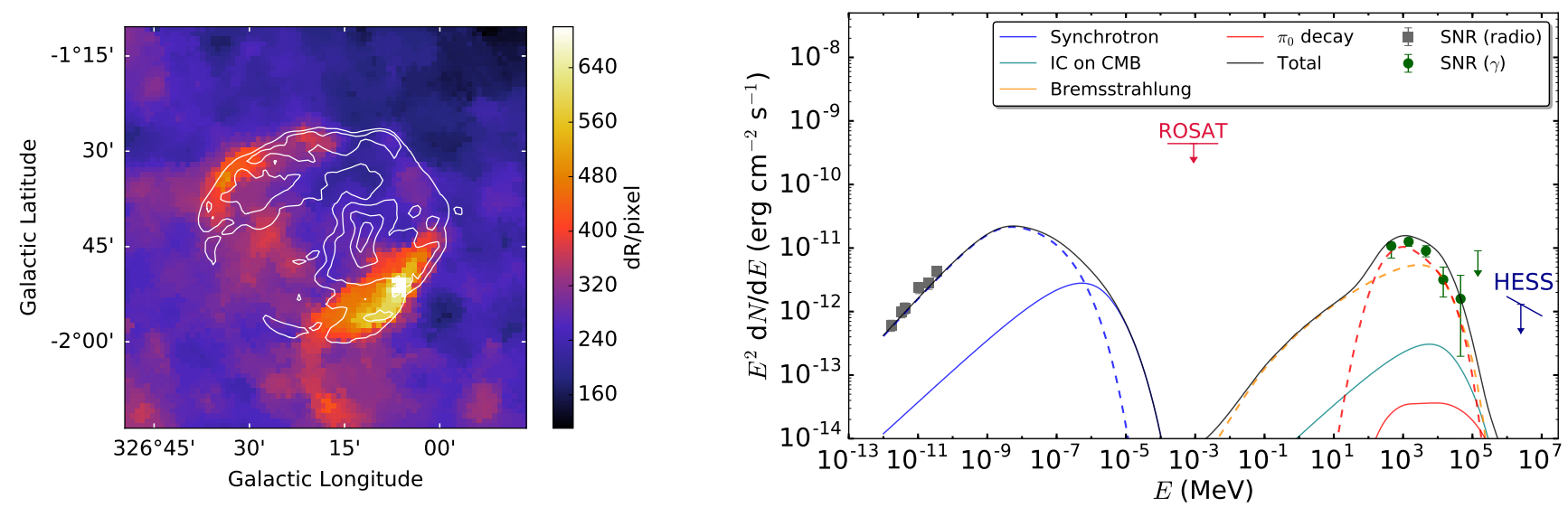

Fig. 10. Left: $\mathrm{H} \alpha$ emission of SNR G326.3-1.8 obtained from the Southern H-Alpha Sky Survey Atlas (Gaustad et al. 2001). The radio contours of the whole SNR are overlaid in white. Right: multiwavelength modeling in the hadronic scenario with two contributions: a main shock (solid lines), and a radiative shock (dashed lines). The radio points are extracted from Dickel et al. (2000), the ROSAT and the H.E.S.S. upper limits come from Kassim et al. (1993) and H.E.S.S. Collaboration et al. (2018) respectively. The values of the parameters are reported in Table 4.

Dennefeld (1980) obtained a spectrum indicating an [S II]/H $\alpha$ ratio characteristic of a radiative shock. This is evidence of the interaction of the shock with neutral material, where some regions of the SNR are entering the radiative phase, while other parts are freely expanding in the ISM. As a consequence, we suggest to model the SNR spectrum with two contributions:

- a radiative shock arising from clouds in the surroundings of the SNR, and 
- a main shock with a velocity of $u_{\mathrm{sh}}=500 \mathrm{~km} \mathrm{~s}^{-1}$ that expands in an ISM density of $n_{0}=0.1 \mathrm{~cm}^{-3}$ (Temim et al. 2013).

Below we calculate the physical parameters associated with the radiative component. Uchiyama et al. (2010) studied the nonthermal emission from crushed clouds in SNRs where reacceleration of preexisting cosmic rays can explain the observed $\mathrm{GeV}$ emission powered by hadronic interactions.

Following this work, the strong shock driven into the clouds has a velocity of

$u_{\mathrm{sh}, \mathrm{cl}}=k \sqrt{\frac{n_{0}}{n_{0, \mathrm{cl}}}} \times u_{\mathrm{sh}}$,

where $k=1.3$ is adopted as in Uchiyama et al. (2010), $n_{0}$ and $n_{0, \mathrm{cl}}$ being the upstream ISM and cloud density, respectively. For the upstream magnetic field in the clouds, we have

$B_{0, \mathrm{cl}}=b \sqrt{\frac{n_{0, \mathrm{cl}}}{\mathrm{cm}^{-3}}} \mu \mathrm{G}$

where $b=v_{\mathrm{A}} /\left(1.84 \mathrm{~km} \mathrm{~s}^{-1}\right)$, with $v_{\mathrm{A}}$ being the Alfvén velocity, the mean value of which is thought to be roughly equal to the velocity dispersion observed in molecular clouds $(\sim 0.5-$ $5 \mathrm{~km} \mathrm{~s}^{-1}$ ), implying $b \sim 0.3-3$ (Hollenbach \& McKee 1989). As in Uchiyama et al. (2010), we assumed that the magnetic pressure in the cooled gas is equal to the shock ram pressure, and we have

$\frac{B_{\mathrm{m}}^{2}}{8 \pi}=k^{2} n_{0} \mu_{\mathrm{H}} u_{\mathrm{sh}}^{2}$,

where $\mu_{\mathrm{H}}$ is the mass per hydrogen nucleus and $B_{\mathrm{m}}$ the downstream magnetic field in the cooled regions

$B_{\mathrm{m}}=\sqrt{\frac{2}{3}} \times\left(\frac{n_{\mathrm{m}}}{n_{0, \mathrm{cl}}}\right) \times B_{0, \mathrm{cl}}$,

with $n_{\mathrm{m}}$ being the downstream density in the cooled regions. In this model, the compressed magnetic field is fixed to $158 \mu \mathrm{G}$ by the pressure in the SNR (Eq. (7)). This requires a large $W_{\mathrm{e}}$ in the clouds for the synchrotron emission to be consistent with the bright observed radio flux. We set $K_{\mathrm{e}-\mathrm{p}}$ in the clouds to 0.03 , which is high, but still reasonable, since a lower $K_{\mathrm{e}-\mathrm{p}}$ would result in an uncomfortably large $W_{\mathrm{p}}$. In any case, the cosmic-ray energy in the shocked clouds must be high. Thus, simultaneously fitting the $\gamma$-ray data and the radio data implies that the compressed density should be relatively low. From Eqs. (6)-(8), we have

$n_{\mathrm{m}}=\sqrt{\frac{3}{\pi \mu_{\mathrm{H}}}} \times \frac{B_{\mathrm{m}}^{2}}{4 b \times u_{\mathrm{sh}, \mathrm{cl}}}$.

We adopted the highest reasonable values $b=3$ and $u_{\text {sh,cl }}=150 \mathrm{~km} \mathrm{~s}^{-1}$ (above which the shock would have no time to become radiative). Thus the downstream density in the cooled regions is $n_{\mathrm{m}}=88.3 \mathrm{~cm}^{-3}$. Taking $n_{0}=0.1 \mathrm{~cm}^{-3}, u_{\mathrm{sh}}=$ $500 \mathrm{~km} \mathrm{~s}^{-1}$ (Temim et al. 2013), $u_{\mathrm{sh}, \mathrm{cl}}=150 \mathrm{~km} \mathrm{~s}^{-1}$ and $b=3$, we obtain for the upstream density and magnetic field in the clouds $n_{0, \mathrm{cl}}=1.88 \mathrm{~cm}^{-3}$ and $B_{0, \mathrm{cl}}=4.11 \mu \mathrm{G}$. This relatively low density is in agreement with the non-detection of $\mathrm{CO}$ lines close to this SNR. The densities encountered in G326.3-1.8 (cloud and intercloud medium) would then be very similar to the Cygnus Loop (Raymond et al. 1988).

The electrons accelerated in the clouds will rapidly cool due to the strong magnetic field in the dense regions, for which we derived the break energy of the particles by equating $\tau_{\text {sync }}=$ $t_{\text {age }} / 2$ (time since the clouds were shocked). At the shock front, the downstream magnetic field is $B_{\mathrm{d}, \mathrm{cl}}=\sqrt{11} B_{0, \mathrm{cl}}=13.6 \mu \mathrm{G}$, assuming a randomly directed field, and we derived the corresponding maximum energy of the particles using $k_{0}=10$ and $u_{\text {sh,cl }}=150 \mathrm{~km} \mathrm{~s}^{-1}$ when equating $t_{\text {acc }}=\min \left\{\tau_{\text {sync }}, t_{\text {age }} / 2\right\}$. For particles trapped in the clouds, we thus find $E_{\mathrm{b}}=15.2 \mathrm{GeV}$ and $E_{\text {max }, \mathrm{e}}=E_{\text {max }, \mathrm{p}}=82.7 \mathrm{GeV}$.

Figure 10 (right) shows the corresponding spectrum with the contributions from the main shock (solid lines) and the radiative shock (dashed lines). With such a high magnetic field and density in the cooled regions, the radiative shock dominates the synchrotron and the $\gamma$-ray emission. Setting $K_{\mathrm{e}-\mathrm{p}}=0.03$, the observed spectrum can be explained with $W_{\mathrm{p}}=1.9 \times 10^{49} \mathrm{erg}$ (and thus $W_{\mathrm{e}}=5.7 \times 10^{47} \mathrm{erg}$ ), corresponding to $3.8 \%$ of $E_{\mathrm{SN}}$ transmitted to the reaccelerated protons in the clouds. To reproduce the radio spectral shape, we used harder indexes for the electrons at the radiative shock $\Gamma_{\mathrm{e}, 1} / \Gamma_{\mathrm{e}, 2}=1.8 / 2.8$, which is also observed in other radiative SNRs (Ferrand \& Safi-Harb 2012) ${ }^{5}$. The $\gamma$-ray cutoff implied by $E_{\text {max,p }}=82.7 \mathrm{GeV}$ fits the observed spectrum well. This is however largely coincidental. $k_{0}$ is unconstrained, $t_{\text {acc }}$ is unknown (we do not know when the clouds were shocked). Uchiyama et al. (2010) predicted an increase in maximum energy by a factor of $\left(\left(n_{\mathrm{m}} / n_{0}\right) / 4\right)^{1 / 3}=2.27$ due to adiabatic compression, which we did not enter into $E_{\max , p}$. The damping of Alfvén waves due to ion-neutral collisions also implies a break in the proton spectrum that we did not take into account because with $B_{0, \mathrm{cl}}=4.11 \mu \mathrm{G}$ and $n_{0, \mathrm{cl}}=1.88 \mathrm{~cm}^{-3}$, it occurs around $100 \mathrm{GeV}$. Observationally, $E_{\text {max,p }}$ must range between 30 and $100 \mathrm{GeV}$, which is in between other radiative SNRs such as W 44 or IC 443 (22 and $239 \mathrm{GeV}$, respectively; Ackermann et al. 2013).

Since our model predicts that radiative shocks can explain the entire spectrum, we cannot assess observational constraints at the main shock. We took $B=10 \mu \mathrm{G}$, implying $B_{\mathrm{ISM}} \approx 3 \mu \mathrm{G}$ (with $r=4$ ), to stay consistent with $B_{0, \mathrm{cl}}>B_{\text {ISM }}$, but $B_{\text {ISM }}$ could have been lower. We also used the typical $10 \%$ of $E_{\mathrm{SN}}$ going into protons, but this and the value of $K_{\mathrm{e}-\mathrm{p}}$ could also be reduced. For the particle spectra, we kept $\Gamma_{\mathrm{e}, 1}=\Gamma_{\mathrm{p}}=2$ and $\Gamma_{\mathrm{e}, 2}=3$ since we have simple acceleration at the main shock and no observational constraints. The corresponding break and maximum energy were calculated following Parizot et al. (2006) with $u_{\mathrm{sh}}=500 \mathrm{~km} \mathrm{~s}^{-1}$ and $k_{0}=10$, as we did for the lepton-dominated scenario. All the values used for the plot are reported in Table 4.

The entire SNR spectrum can thus be explained by the emission from radiative shocks. Although there is no clear correlation between the $\mathrm{H} \alpha$ and the radio maps, this difference can be explained by the orientation of the magnetic field: where $B$ is perpendicular to the shock velocity, the synchrotron emission is highest (compression of the tangential component of the field), whereas optical emission should be enhanced when $B$ is parallel since the compression is no longer limited by the magnetic field. Quantitatively, the total energy required in the cosmic rays at the radiative shocks is high. Assuming $20 \%$ of the pressure in the radiative shocks is in the form of cosmic rays (the rest is mostly magnetic), it requires a surface covering factor close to $50 \%$ (consistent with the fact that we see little deviation from a uniform disk). This may be tested by deep $\mathrm{H} \alpha$ imaging.

\footnotetext{
5 Radio spectral indexes of some radiative SNRs, such as W 44 or IC 443, can be found at http: //www . physics . umani toba.ca/snr/ SNRcat/
} 


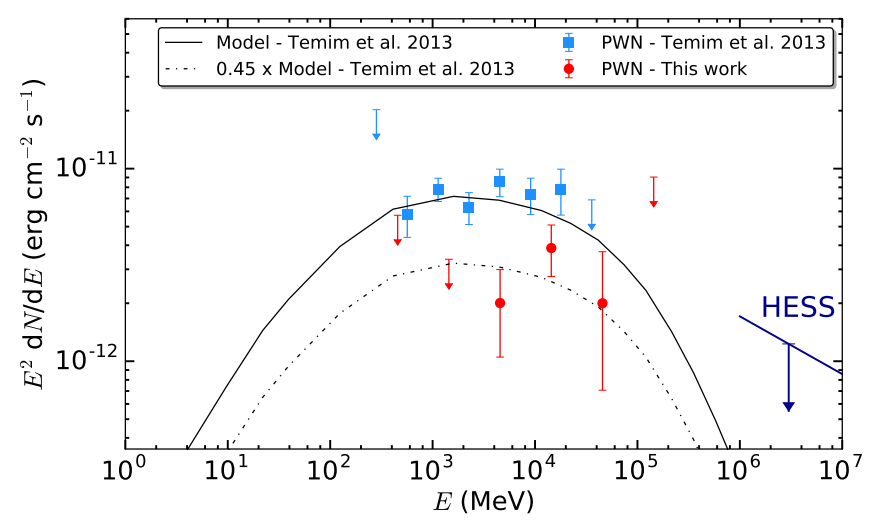

Fig. 11. Comparison of the $\gamma$-ray PWN spectra where the model derived in Temim et al. (2013) is multiplied by a factor of 0.45 to fit our data.

\subsection{PWN spectrum}

We find that the largest portion of the $\gamma$-ray emission comes from the SNR, presumably from the hadronic process. Nevertheless, the PWN appears to contribute as well. We briefly and qualitatively discuss the effect of the PWN flux diminution on the physical parameters derived in Temim et al. (2013), who assumed that the entire $\gamma$-ray emission originated in the PWN, and based their analysis on the previous data release (Pass 7). Figure 11 compares the two $\gamma$-ray spectra, where the model of Temim et al. (2013), who assumed a fully leptonic origin of the emission, is scaled to fit our data. The current flux corresponds to $45 \%$ of the previous one.

If we approximate $W_{\mathrm{e}} \approx \int_{0}^{t_{\text {age }}} \dot{E} \mathrm{~d} t, \dot{E}$ being the energy loss rate of the pulsar, we obtain

$W_{\mathrm{e}} \approx \dot{E}_{0} \frac{\tau_{0} t_{\mathrm{age}}}{\tau_{0}+t_{\mathrm{age}}}$

where $\tau_{0}$ is the initial spin-down timescale of the pulsar, and $\dot{E}_{0}$ is the initial spin-down power. Temim et al. (2013) derived $\tau_{\mathrm{c}} \approx 2.1 \times 10^{4} \mathrm{yr}$ and $\dot{E}_{0}=3 \times 10^{38} \mathrm{erg} \mathrm{s}^{-1}$. We now require less than half of $W_{\mathrm{e}}$, leading to $\dot{E}_{0}=1.35 \times 10^{38} \mathrm{erg} \mathrm{s}^{-1}$ for the same age and initial spin-down time scale of the pulsar.

In their 1D model, the observed SNR radius is reached at an age of $19 \mathrm{kyr}$, for which they estimated the PWN magnetic field to be $B_{\mathrm{PWN}}=34 \mu \mathrm{G}$. The decrease in $W_{\mathrm{e}}$ would thus also imply a higher magnetic field to still stay consistent with the radio flux of the PWN.

However, a more nuanced interpretation is required given the complexity of this object. This will require more investigations and detailed modeling which are beyond the scope of this paper. We also note that the PWN spectrum derived in this analysis is model dependent when considering the assumption made for the SNR. In any case, its flux is reduced compared to previous studies since the SNR contributes most of the $\gamma$-ray emission.

\section{Conclusions}

We performed an analysis from $300 \mathrm{MeV}$ to $300 \mathrm{GeV}$ of the composite SNR G326.3-1.8 with the Fermi-LAT Pass 8 data. We took advantage of the new PSF3 event class by selecting the events with the best angular reconstruction to limit mixture between the SNR and the PWN contributions and also emission from the Galactic plane. Using the pointlike and the gtlike frameworks, we confirm that the emission is significantly extended (more than $13 \sigma$ ) between $300 \mathrm{MeV}$ and $300 \mathrm{GeV}$. We performed an analysis in five energy bands, which showed that the morphology evolves with energy and the size shrinks toward the radio $\mathrm{PWN}$ at high energies $(E>3 \mathrm{GeV})$. We thus investigated a more detailed morphology using the radio map of the PWN as a starting point. We find that it is clearly not sufficient to describe the $\gamma$-ray data and that an additional extended component is needed. We then tested different models for an additional contribution such as a uniform disk, the radio map of the remnant, and its homogeneously filled radio template, called here the SNR mask. Using the maximum likelihood fitting procedure starting at $1 \mathrm{GeV}$, we find that the model with the SNR mask and the radio PWN reproduces the $\gamma$-ray emission best.

Modeling both $\gamma$-ray emissions by a power law from $300 \mathrm{MeV}$ to $300 \mathrm{GeV}$, we obtained a spectral separation between the two components: a softer spectrum for the remnant $(\Gamma=2.17 \pm 0.06)$, and a harder spectrum for the nebula $(\Gamma=1.79 \pm 0.12)$. The corresponding SEDs also highlight their different contributions: the SNR dominates the low-energy part $(300 \mathrm{MeV}-10 \mathrm{GeV})$, while the PWN protrudes at higher energies $(E>10 \mathrm{GeV})$.

Concerning the PWN spectrum, we briefly discussed the effect of the flux diminution (about 55\%) compared to previous studies, which assumed that the entire $\gamma$-ray emission may come from the PWN.

The spectral modeling of the SNR emission disproves the leptonic scenario since it requires an unrealistic high energy budget in the electrons to fit the $\gamma$-ray data $\left(W_{\mathrm{e}}\right.$ of several $10^{49} \mathrm{erg}$ ). As $\mathrm{H} \alpha$ emission has been reported in this SNR, we suggest a spectral modeling where the main contribution arises from regions entering the radiative phase. The high magnetic field and density in the cooled regions lead to enhanced synchrotron and $\mathrm{GeV}$ emission that dominates the entire spectrum. The bestfit model involves a compressed magnetic field of $10 \mu \mathrm{G}$ and $158 \mu \mathrm{G}$ at the main and the radiative shock, respectively. With $3.8 \%$ of the kinetic energy released by the supernova ending into accelerated particles at the radiative shock, we find that an electron-proton ratio of $K_{\mathrm{e}-\mathrm{p}}=0.03$ can adequately reproduce the observed spectrum. Although this ratio is slightly higher than one would expect, this is the most appropriate and consistent model we find that can simultaneously explain the high radio and $\gamma$-ray emissions from this SNR. In the future, the Cherenkov Telescope Array (CTA) will give more insight into the properties of this source, since it will provide better sensitivity above $30 \mathrm{GeV}$.

Acknowledgements. We thank D. Castro and the referee P. Slane for their helpful comments on this paper. The Fermi LAT Collaboration acknowledges generous ongoing support from a number of agencies and institutes that have supported both the development and the operation of the LAT as well as scientific data analysis. These include the National Aeronautics and Space Administration and the Department of Energy in the United States, the Commissariat à l'Energie Atomique and the Centre National de la Recherche Scientifique / Institut National de Physique Nucléaire et de Physique des Particules in France, the Agenzia Spaziale Italiana and the Istituto Nazionale di Fisica Nucleare in Italy, the Ministry of Education, Culture, Sports, Science and Technology (MEXT), High Energy Accelerator Research Organization (KEK) and Japan Aerospace Exploration Agency (JAXA) in Japan, and the K. A. Wallenberg Foundation, the Swedish Research Council and the Swedish National Space Board in Sweden. Additional support for science analysis during the operations phase is gratefully acknowledged from the Istituto Nazionale di Astrofisica in Italy and the Centre National d'Études Spatiales in France. This work performed in part under DOE Contract DE-AC02-76SF00515. We also acknowledge the Southern HAlpha Sky Survey Atlas (SHASSA), which is supported by the National Science Foundation.

\section{References}

Acero, F., Ackermann, M., Ajello, M., et al. 2015, ApJS, 218, 23 Acero, F., Ackermann, M., Ajello, M., et al. 2016a, ApJS, 223, 26 
Acero, F., Ackermann, M., Ajello, M., et al. 2016b, ApJS, 224, 8

Ackermann, M., Ajello, M., Allafort, A., et al. 2013, Science, 339, 807

Ackermann, M., Ajello, M., Atwood, W. B., et al. 2016, ApJS, 222, 5

Ackermann, M., Ajello, M., Baldini, L., et al. 2017, ApJ, 843, 139

Atwood, W., Albert, A., Baldini, L., et al. 2013, 2012 Fermi Symp. proc. - eConf C121028 [arXiv:1303.3514]

Bell, A. R. 1978, MNRAS, 182, 147

Castelletti, G., Dubner, G., Brogan, C., \& Kassim, N. E. 2007, A\&A, 471, 537

Dennefeld, M. 1980, PASP, 92, 603

Dickel, J. R., Milne, D. K., \& Strom, R. G. 2000, ApJ, 543, 840

Ferrand, G., \& Safi-Harb, S. 2012, Adv. Space Res., 49, 1313

Gaustad, J. E., McCullough, P. R., Rosing, W., \& Van Buren, D. 2001, PASP, 113,1326

Goss, W. M., Radhakrishnan, V., Brooks, J. W., \& Murray, J. D. 1972, ApJS, 24, 123

H.E.S.S. Collaboration, Abdalla, H., Abramowski, A., et al. 2018, A\&A, 612, A3

Hollenbach, D., \& McKee, C. F. 1989, ApJ, 342, 306

Kassim, N. E., Hertz, P., \& Weiler, K. W. 1993, ApJ, 419, 733
Kerr, M. 2010, PhD Thesis, University of Washington, USA

Mills, B. Y., Slee, O. B., \& Hill, E. R. 1961, Aust. J. Phys., 14, 497

Milne, D. K. 1971, Aust. J. Phys., 24, 429

Milne, D. K., Goss, W. M., Haynes, R. F., et al. 1979, MNRAS, 188, 437

Parizot, E., Marcowith, A., Ballet, J., \& Gallant, Y. A. 2006, A\&A, 453, 387

Plucinsky, P. P. 1998, Mem. Soc. Astron. It., 69, 939

Raymond, J. C., Hester, J. J., Cox, D., et al. 1988, ApJ, 324, 869

Rosado, M., Ambrocio-Cruz, P., Le Coarer, E., \& Marcelin, M. 1996, A\&A, 315, 243

Temim, T., Slane, P., Castro, D., et al. 2013, ApJ, 768, 61

Uchiyama, Y., Aharonian, F. A., Tanaka, T., Takahashi, T., \& Maeda, Y. 2007, Nature, 449, 576

Uchiyama, Y., Blandford, R. D., Funk, S., Tajima, H., \& Tanaka, T. 2010, ApJ, 723, L122

van den Bergh, S. 1979, ApJ, 227, 497

Whiteoak, J. B. Z., \& Green, A. J. 1996, A\&AS, 118, 329

Zabalza, V. 2015, in 34th International Cosmic Ray Conference (ICRC2015), 34, 922 